\section{Novel therapeutic developments in acute myeloid and acute lymphoblastic leukemia}

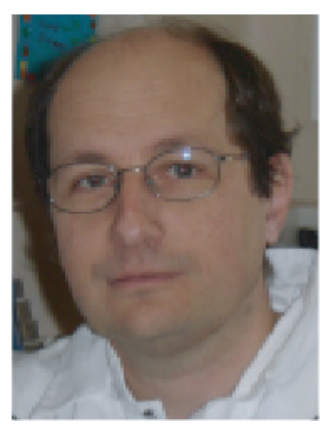

Xavier Thomas* is in charge of the management and treatment of adult patients with acute myeloid leukemia and acute lymphoblastic leukemia in the Department of Hematology at the Lyon-Sud Hospital (France). He is member of the American Society of Hematology (ASH), American Society of Clinical Oncology (ASCO), American Association for Cancer Research (AACR) and European Hematology Association (EHA). He is actively involved in the Group for Research in Adult Acute Lymphoblastic Leukemia (GRAALL) and the Acute Leukemia French Association (ALFA). He has published more than 300 peerreviewed papers and has served as a referee for several journals, such as Blood, Leukemia, The Lancet, Leukemia and Lymphoma, and Cancer.
Q What influenced you to go into a career in hematology \& specifically leukemia?

As for many people, there is always a good reason for going into a career in the field of oncology. My father died from glioblastoma when I was a medical student. Until that point, I was expecting a career as a cancer researcher. Several years later, I started my medical residency in the leukemia unit at the Edouard Herriot Hospital in Lyon (France). Direct contact with patients and the close relationship between clinic and biology in this field led me then turn my attention to acute leukemia.

\section{Q Who would you say has had the greatest influence on your education \& career?}

My mentor was Professor Eric Archimbaud with whom I participated to the development of many clinical trials in acute myeloid leukemia and to the development of biological research on adhesion molecules. $\mathrm{He}$ also involved me in the organization of the 1987 meeting of the International
Society of Experimental Hematology that held in Cannes (France). After his sudden death in a car accident in 1988, I took the charge of the leukemia program in Lyon and tried to keep going with the engagements he took all along his career and the program he initiated on the use of timedsequential chemotherapy and 'priming' in acute myeloid leukemia. I have also been greatly influenced by Professor Denis Fiere, who was, in the 1990s, head of the Hematology Department in Lyon and leader of the French group for treatment of adult acute lymphoblastic leukemia. He gave me the opportunity to conduct and publish the LALA-94 trial, which was at that time one of the most important published series in adult acute lymphoblastic leukemia. After residency, I also have had the great fortune to work for 6 months with Professors Jean Klasterski and Françoise Meunier at the Jules Bordet Institute in Brussels, and as a postdoc, I spent 2 years on myeloma research in Professor Josh Epstein's laboratory at the Arkansas Cancer Research Center in Little Rock.
*Hospices Civils de Lyon, Lyon-Sud Hospital, Hematology, Bat.1G, 165 chemin du Grand Revoyet, 69495 Pierre Benite cedex, France; Tel.: +33 478862235; Fax: +33 472678880; xavier.thomas@chu-lyon

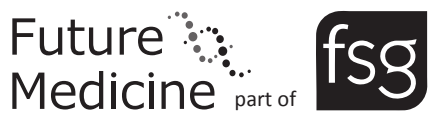

\author{
KEYWORDS \\ - acute lymphoblastic leukemia \\ - acute myeloid leukemia \\ - targeted therapy
}


Q You are currently in charge of the Leukemia Treatment Program at the Lyon-Sud Hospital in France. Can you please tell our readers about this program \& your specific responsibilities?

Our current program can be divided into clinical research and biological research. My main research activity is to develop clinical research on acute leukemia at Lyon-Sud Hospital. We effectively moved few years ago from Edouard Herriot Hospital in the center of the city to this new hospital in the south of the city, where was already installed one division of hematology focused on the treatment of lymphomas and myeloma. Our current department, under the head of Professor Gilles Salles, is therefore one of the biggest in Europe with six units of hospitalization and two day care units. Clinical research in which I am involved comprises academic trials testing mainly therapeutic strategies and trials from the industry testing investigational drugs in adult acute myeloid leukemia or acute lymphoblastic leukemia. We are participating to the French ALFA and GRAALL academic groups for acute myeloid leukemia and acute lymphoblastic leukemia, respectively. Novel therapeutic development includes our participation in many trials from the industry. Our clinical research unit is particularly developed with overall 17 clinical research associates with a training of nurses and others with a $\mathrm{PhD}$ degree. We have currently 15 opened trials for acute leukemia. We are then participating to trials involving bromodomain inhibitors, polokinase inhibitors, hypomethylating agents, new formulation of L-asparaginase, ponatinib, anti$\mathrm{Bcl} 2 \ldots$ in acute myeloid leukemia, and mainly trials involving monoclonal antibodies (blinatumomab, inotuzumab ozogamicin, epratuzumab) in acute lymphoblastic leukemia.

Q You recently authored an article published in the European Journal of Hematology

on the topic of acute myeloid leukemia in the pregnant patient. What challenges are associated with designing a treatment program for pregnant patients?

There is no standard approach and no specific treatment program for this clinical dilemma. That is the reason why writing a review on this subject was necessary. Hopefully acute leukemia in the pregnant woman represents a rare entity. The diagnosis of acute leukemia during pregnancy places the patient and her family into a severe conflict requiring the medical personnel support and professional accompaniment. Actually, each case should be examined individually, considering both the aggressiveness of leukemia and the stage of the pregnancy when the therapy is applied. Generally, pregnant women in the first trimester should be offered termination of pregnancy because of the potentially fetal consequences of chemotherapy and the maternal complications of leukemia. Chemotherapy treatment during the second to third trimester may not require termination as remission of acute leukemia and delivery of a normal infant seem likely. However, fetal surveillance and monitoring for adequate growth is absolutely required. It is essential to provide the pregnant woman with a multidisciplinary team of physicians including hematologists, but also gynecologists, neonatologists and obstetricians as well as psychologists. Anyway, every decision about treatment must be case specific and should be made together with the patient after consideration of risks and benefits.

\section{Q Can you please tell our readers about} any specific research you are working on currently?

As I already told my main research field is clinical research. However, I also participate to biological researches in the setting of the INSERM unit 590 directed by Professor Charles Dumontet and in collaboration with researchers from different laboratories. Chemoresistance of acute myeloid leukemia cells with disease repopulating potential remains one of the most important challenges in acute myeloid leukemia. Our last research concerns the relevance of the CD34/CD38 subpopulations in the outcome of patients with acute myeloid leukemia. A large $\mathrm{CD} 34^{+} \mathrm{CD} 38^{-}$immature cell population at the time of diagnosis has been correlated with a higher percentage of chemotherapy-resistant cells and minimal residual disease. The initial aim of our study was to analyze the importance of the $\mathrm{CD} 34^{+} \mathrm{CD} 38^{-}$cell population when compared with the $\mathrm{CD} 34^{+} \mathrm{CD} 38^{+/ \text {low }}$ and $\mathrm{CD} 34^{+} \mathrm{CD} 38^{+1}$ high leukemic cell subpopulations and to determine its correlations with known prognostic factors and leukemia characteristics, as well as with response to therapy and survival. The further step will be to compare flow sorter-purified genetically characterized acute myeloid leukemia stem and progenitor cell populations of identical immunophenotype from patients at diagnosis 
and following chemotherapy. Comparative analysis of these cell populations will be performed to determine the cell cycle status of chemotherapy-resistant malignant stem/progenitor cells, the transcriptome of chemo-resistant malignant stem/progenitor cells and drug classes that provide efficacy on these cells.

\section{Q In your opinion, will targeted therapy be} the way forward for cancer treatment?

It is clear now that additional gains are unlikely to be achieved by simply intensifying therapy further. There are few interesting new cytotoxic agents and anyway intensification has reached its limits due to the offset of unacceptable toxicities. There are new procedures for allogeneic stem cell transplantation, but they modestly improve the outcome of adult acute leukemia, and unfortunately introduce higher risks of acute and late complications. There is therefore a clear and compelling rationale for developing new therapies that specifically target the molecular abnormalities that cause leukemia. More and more points of intervention are being identified that may respond to targeted drugs. The biology of leukemia stem cell begins also to be better understood. This is particularly important since the relevant goal of leukemia therapy remains the eradication of leukemia stem cells. Targeting the leukemia stem cell will definitively open a new era of treatment in acute leukemia. Over the next years, we will certainly be involved in more and more clinical trials testing newtargeted therapies, some of which are now just starting to emerge. I hope that we will be able to combine those drugs and to include them in conventional chemotherapy backbone to design new therapeutic strategies. Of course there is still much work to do, particularly in identifying the optimal combinations and scheduling of these novel agents. So there are very exciting times ahead for the current young hematologists.

\section{Q What are the major breakthroughs in the treatment of leukemia that you have witnessed in your career?}

The past two decades have yielded remarkable improvements in long-term outcomes for patients with acute leukemia. A better understanding of the biology of this disease, the utilization of riskadapted therapy, the development of protocols with optimized combinations of cytotoxic drugs, postinduction intensifications including allogeneic stem cell transplantation, effective CNS prophylaxis and a prolonged maintenance therapy have significantly raised long-term survival rate for pediatric acute leukemia. Although giving less spectacular results, the same therapeutic approaches have been applied to adults, leading to an improved response rate and survival. My generation has therefore witnessed many progresses in the treatment of leukemia. Bone marrow transplantation, which became of practical value in the 1970s, has continued its development over the last decade. Myeloablative chemotherapy could be carried out more easily and safely with hematopoietic rescue using peripheral blood stem cells. To get around the age restriction of allogeneic stem cell transplantation, a number of investigators have developed lessintensive programs that rely less or not at all on chemo radiation therapy for tumor eradication and shift the burden of tumor cell kill toward the graft-versus-leukemia effect. The demonstration of the presence of hematopoietic stem cells in cord blood has suggested the use of these cells for transplantation. Investigators began also to explore the possible antileukemic effect of donor lymphocyte infusions for patients who relapsed after transplantation. Concomitantly, significant advances also came from the development of clinical research. Collaborative groups of clinical investigators were formed worldwide, and major public programs of research support for leukemia therapy were established. Improvements of supportive care also played a major role in outcome improvements. This involved advances in terms of blood transfusion, but also the introduction of the recombinant hematopoietic growth factors.

But all these therapeutic advances were progressively obtained overtime. Actually, I have witnessed in my career two real major breakthroughs. The first one and the most unexpected to my opinion was the therapeutic advance in the treatment of acute promyelocytic leukemia. The introduction of all-trans retinoic acid in the treatment of acute promyelocytic leukemia at the end of the 80 s really impressed me as a young resident. I remember the first patient we treated in Lyon entering in complete remission just with vitamins and without any complications such as coagulopathy, while we were previously regularly confronted with intensive chemotherapy to dramatic complications such as bleedings responsible of early deaths in this type of patients. Furthermore, molecular technology has shown that acute promyelocytic leukemia was a result of a translocation involving the retinoic acid receptor gene on 
chromosome 17 and the $P M L$ gene on chromosome 15 , suggesting a simple explanation of the molecular mechanism of action. Initial studies of all-trans retinoic acid and its combination with chemotherapy completely changed the prognostic of the disease and transformed the disease from one that was initially highly fatal to one that is now highly curable. The treatment of acute promyelocytic leukemia also benefited from another important molecule, arsenic trioxide. Arsenic has been an active ingredient, since ancient times, in the folk remedies and organic arsenicals were once a mainstay of cancer chemotherapy. During the last decades, publications from China established a remarkable efficacy of arsenic trioxide as a single agent for the treatment of acute promyelocytic leukemia and signed the rebirth of arsenic therapy. The introduction of this treatment was not felt as important as that of retinoic acid, but the recent publication by Francisco Lo-Coco of a schedule combining all-transretinoic acid and arsenic trioxide in first-line therapy represents certainly a new step forward in the treatment of this subtype of leukemia. This study established a new standard front-line treatment, without the use of cytotoxic chemotherapy, at least in acute promyelocytic leukemia patients with standard-risk disease.

The second breakthrough was the introduction of tyrosine kinase inhibitors, starting with imatinib mesylate, in the treatment of Philadelphia chromosome-positive leukemias. In Europe, imatinib really changed our vision of the treatment in chronic myeloid leukemia with our participation to the STI571 0106 trial, which randomized this new agent with the combination of interferon- $\alpha$ and cytarabine and started few months after Brian Druker's presentation in 1999 at ASH meeting in New Orleans. Imatinib mesylate also involves a relatively simple mechanism of action by blocking the kinase activity of the $A B L$ proto-oncogene. For sure, the application of tyrosine kinase inhibitors in the treatment of Philadelphia chromosomepositive acute lymphoblastic leukemia appeared later and the place of tyrosine kinase inhibitors in combination with chemotherapy took longer to be defined, and is still under investigation. However, Philadelphia chromosome-positive acute lymphoblastic leukemia, which was historically known for its very poor outcome, is now seen totally differently, particularly for elderly patients for whom allogeneic transplant cannot be considered.
Q Finally, how do you see this highly dynamic field developing over the next 5-10 years?

It is clear that hematology remains a leading field among oncology for the application and development of new therapeutics. However, patients will still need to be treated with a combination of therapeutic agents. It is unlikely to found other agents, which can revolutionize the treatment for acute myeloid leukemia or acute lymphoblastic leukemia, as it was the case with all-trans retinoic acid or imatinib for acute promyelocytic leukemia and chronic myeloid leukemia, respectively. Since acute leukemia is a collection of molecularly heterogeneous diseases, further improvements should depend on the development of new strategies according to a better knowledge of the disease. Each agent would be expected to be effective on one or few subsets of molecular abnormalities involved in the leukemic process and the combination, sequentially or concurrently, of these agents could potentiate their effects to eradicate the disease. We should keep in mind lessons from the past. Major improvements in the treatment of acute leukemia followed the development of 'Total therapy' in the 1960s. This resulted in a tremendous increase in the survival rate of patients with acute lymphoblastic leukemia and gave the base of the treatment currently used in first-line therapy. The principles that were applied took into account the advantage of combination chemotherapy in overcoming initial drug resistance and inhibiting acquired resistance, as well as the superiority of some drugs for remission induction and others for its continuation. We should take advantage of this example and of the tremendous step forward done in the knowledge of leukemia biology over the past decades to design similar approaches involving and taking advantage of all new agents and therapeutic strategies currently available. The introduction of targeted therapy should also develop personalized treatments. Equally important will be the development of new objective criteria with which to define response to therapy. The notion of cytological remission does not correspond anymore to the current need. We should refine the definition of response to therapy and take into account molecular response to monitor the evolution of the disease and guide eventual modifications in therapeutics based on progression of the disease. Therapeutic strategies should also take into account refinement of the definitions of specific subclasses of this disease to define aggressiveness of the disease and adapt intensity and specificity of treatments according to the prognosis. A variety of approaches 
to immunotherapy are also currently being investigated. Optimizing the effectiveness of immunotherapy will require targeting the antileukemia immune response at multiple levels, which may be achieved through synergistic combinations. Exciting advances include adoptive transfer of chimeric antigen receptor T cells. However, many challenges remain if durable malignant cell eradication with minimal toxicity is to be achieved. Finally, a better understanding of the biology of the leukemia stem cells will certainly emerge as a fundamental point since eradication of these cells (as opposed to the reduction in the number of bulk leukemia cells) remains the relevant goal of leukemia therapy.

\section{Disclaimer}

The opinions expressed in this interview are those of the interviewee and do not necessarily reflect the views of Future Medicine Ltd.

\section{Financial \& competing interests disclosure}

$X$ Thomas has no relevant affiliations or financial involvement with any organization or entity with a financial interest in or financial conflict with the subject matter or materials discussed in the manuscript. This includes employment, consultancies, honoraria, stock ownership or options, expert testimony, grants or patents received or pending, or royalties.

No writing assistance was utilized in the production of this manuscript. 